\title{
Experimental analysis of the tool-to-workpiece vibrations transmission during chatter development in milling processes
}

\begin{abstract}
Eduardo Rubio
Universidad Autónoma de Aguascalientes, Centro de Ciencias de la Ingeniería, Mahatma Gandhi 6601, Col. El Gigante, Ejido Arellano, Aguascalientes, Ags. C.P. 20340, México. E-mail: erubio@correo.uaa.mx. Tel: +52 (449) 9107400/9510.

Accepted 14 September, 2012

An experimental work to analyze the mechanical vibrations that arise during chatter development in milling processes was conducted. The tool vibrations and the frequencies that are excited at the workpiece due to the tool impacts were studied for various levels of chatter. Tool vibrations were measured with an inductive sensor, while the workpiece vibrations were measured with an accelerometer. The relevance of the different vibrations measurement types, named acceleration, velocity and displacement, were investigated in relation to the information that each variable offers for chatter analysis. Experimental results showed that tool and workpiece frequencies are well localized in the frequency spectra. Velocity measurements reported tool and workpiece vibrations adequately, and this variable is a good indicator of chatter development.
\end{abstract}

Key words: Milling, chatter development, inductive sensor, accelerometer, vibrations transmission.

\section{INTRODUCTION}

Chatter vibration is a problem that may arise during machining operations. It is a vibration of the cutting tool relative to the workpiece and it can increase manufacturing costs, reduce the tool life, produce low quality surfaces and early failure of the cutting tool (Khraisheh et al., 1995). Chatter in milling is produced when the steady forced excitation of the teeth impacting the workpiece is modulated into vibration near the natural frequency of the system. This is due to a feedback mechanism present in milling when a variable cutting force, that is proportional to the chip thickness during the material removal, affects the tool vibration producing a phenomenon known as regeneration of waviness (Schmitz and Smith, 2012).

The stability of the process is dependent on the machine-tool-workpiece system which can be expressed in terms of the frequency response function (FRF) of the system tool tip. Vibration modes can be identified and higher modal frequencies are excited as milling speed increases. FRF at the tool tip is helpful in chatter stability analysis (Tang et al., 2009).

Different approaches to develop techniques to improve industrial machining conditions can be found in the literature. All of them are based in single or multi-sensor data acquisition systems. The use of acoustic signals to detect chatter is a common practice according to Axinte et al. (2004), where acoustic emission, vibration and cutting force sensors are used to correlate the quality of machined surfaces. It was demonstrated that vibration signals were sensitive to detect chatter marks, while the acoustic emission signal was efficient for the detection of surface anomalies. Acoustic signals have also been used to establish control strategies according to Tsai et al. (2010). In this work, a microphone signal was used to obtain a dimensionless index based on an exponential function. This function takes into consideration the output voltage of the sensor for speed compensation of the spindle speed in a non-invasive real-time chatter prevention technique.

Research works can be found to study the relationship among cutting conditions, tool deflection, cutting forces and the generated sound from the process according to Bagci (2011). In this work, two precision inductive displacement sensors placed at $90^{\circ}$ were used for cu tter deflection measurement in surface milling. Results for 


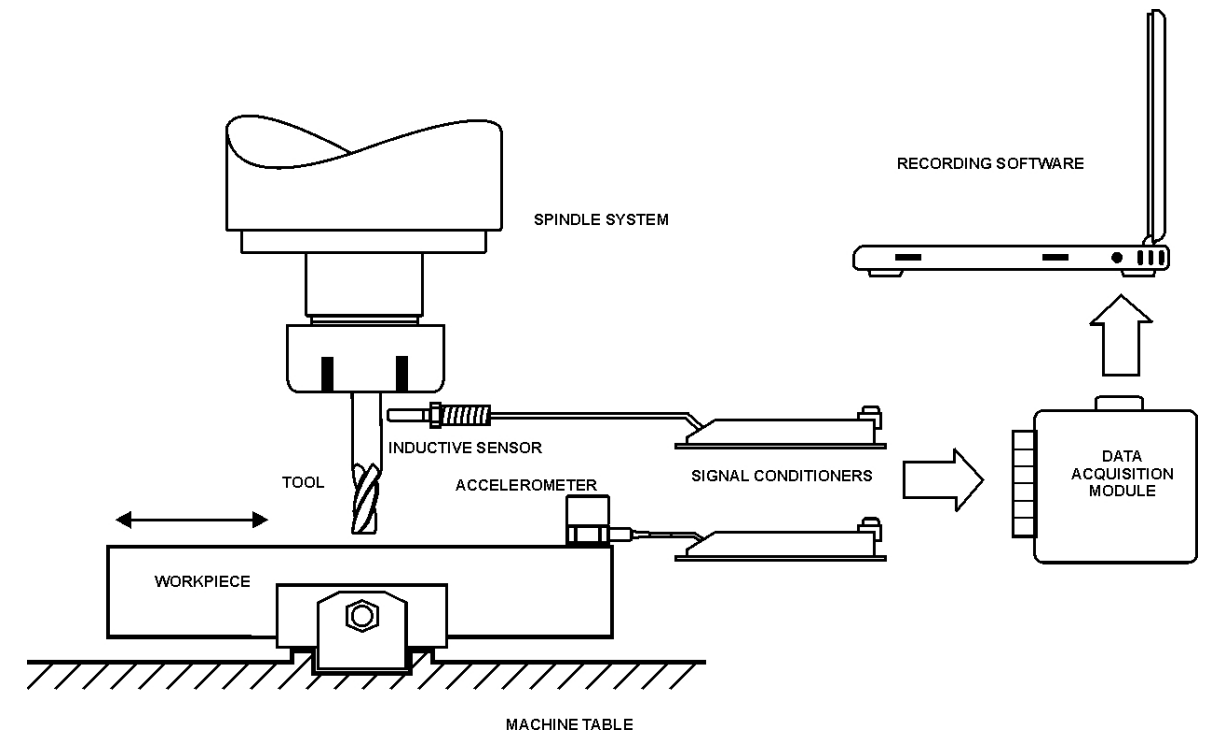

Figure 1. Experimental set-up for milling experiments.

different feed rates were used to correlate inductive sensor information and the acoustic signal, showing that sound pressure level is a viable method for process monitoring.

Uncommon methods, such as those that use laser displacement sensors, can be found for cutting tool diagnostics. Ryabov et al. (1998) showed the advantages of this technique in milling processes. Their work demonstrated the feasibility of various applications, namely tool setting, cutter geometry, tool failure, cutter deterioration, and chatter detection. A scanning signal of the rotating tool was obtained offering detailed information. Special experimental and processing techniques had to be implemented to prevent metal chips from hitting the sensor and to extract information from a noisy signal when coolant was present. Chatter patterns were extracted from the tool vibration signals. Capacitance displacement sensors are rarely found as cutting force sensing methods. Albrecht et al. (2005) showed a work intended to demonstrate compensation techniques that enable this kind of sensors to be used in high frequency bandwidth cutting in milling. The capacitance sensor was installed on the main spindle of the machine and the bandwidth of the sensor system was increased from 350 to $1000 \mathrm{~Hz}$.

The frequency analysis is commonly used as a vibration analysis tool and harmonic and modulation effects may arise in the spectrum. They may appear both, as low and high frequency components. This can be shown in the spectrum obtained by Suh et al. (2002) for unstable milling operations, where harmonics of the cutting frequency and other higher modes of vibration are excited when chatter is present. Insperger et al. (2003) stated that there are several frequency sets arising from the vibration signals that make the vibration analysis not trivial. Their power spectra in milling tests showed that the tooth pass excitation frequency with higher harmonics within a wide portion of the spectrum, and Hopf type or period double type frequencies, among others.

These harmonics can also be observed in finish milling of hardened steels according to Toh (2004). The vibration analysis of the experiments performed with a worn cutter showed the fast Fourier transform (FFT) frequency analysis with a dominant peak corresponding to the tooth passing frequency (TPF). Subsequent spectral lines could be identified as the harmonics of this frequency. Additionally, small peaks were observed around the TPF that were associated to the radial deflection of the cutter and its modulation due to chip thickness variation. Tatar and Gren (2008) found similar results and showed results where harmonics are classified as the spindle rotation order. In this case, peaks for cutting force measurements appeared up to 6TPF, while for the spindle vibration, it could be identified up to 9TPF. A spectrum of the vibrations with many peaks above the 14th spindle rotation order, attributed to the machine bearings, was obtained.

Periodic and stationary vibration signals for milling under stable conditions have been reported (Kuljanic et al., 2008). For these conditions, the peaks of the spectra were located at integer multiples of the spindle revolution frequency. However, some anomalies or chatter peaks were present when the system operated under unstable conditions. These peaks were located at the resonance frequency of the spindle-cutter mechanical system.

\section{MATERIALS AND METHODS}

The set-up shown in Figure 1 was used for the milling experiments. Milling runs were carried out in a Minimill HAAS 3-axis vertical 

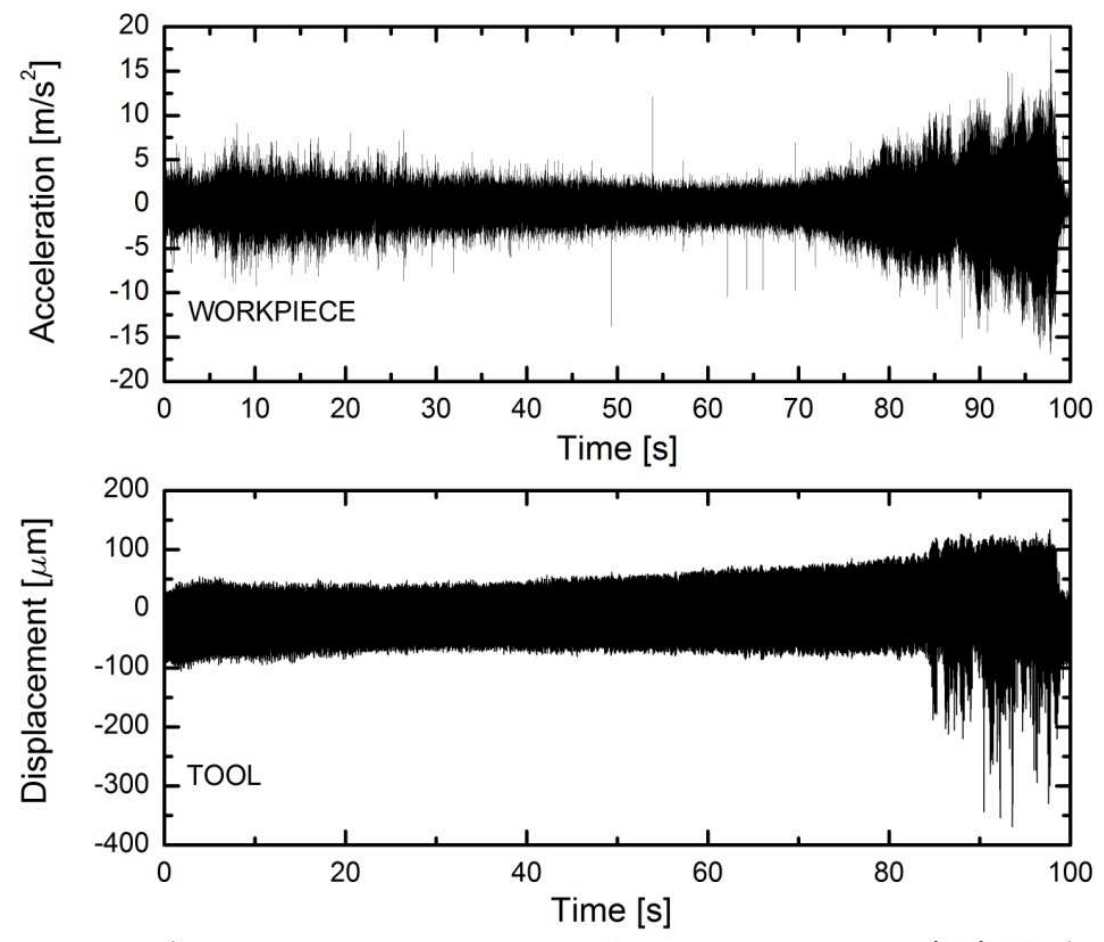

A

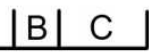

A - No chatter B-Moderate chatter C- Severe chatter

Figure 2. Workpiece and tool vibrations amplitude.

machining center, equipped with a four fluted 3/8" diameter high speed steel milling cutter. The tool tip displacement was measured with an ENTEK 2109 non-contact precision inductive pickup and workpiece vibrations with a KISTLER $8712 \mathrm{~A}$ accelerometer. Impact testing was performed with a Kistler 9724A impact hammer. A National Instruments NI USB-6251 data acquisition was used to record the information in a portable personal computer at a rate of $100 \mathrm{MS} / \mathrm{s}$. Additionally, hardware conditioning of the acceleration signal was performed to obtain the velocity and displacement amplitude of the vibrations. Rectangular blocks of cold rolled steel were used as test pieces. Test pieces were clamped to the machining center at an angle of $1.5^{\circ}$ to gradually in crease the depth of the cut, and experiments were conducted until the characteristic sound produced by severe chatter was evident.

\section{RESULTS AND DISCUSSION}

The workpiece and tool vibrations graphs in time-domain are as shown in Figure 2. Since the workpiece was installed with a small angle with respect to the horizontal, the cutting depth gradually increased until the machining conditions entered the unstable state. As expected, near the end of the plot, when chatter began, the workpiece was excited in such a form that the vibrations amplitude grew more pronouncedly. Three major regions have been highlighted to depict different machining conditions. Region $\mathrm{A}$ is characterized by the absence of chatter, while regions $\mathrm{B}$ and $\mathrm{C}$ correspond to moderate and severe chatter, respectively. The inductive sensor, located next to the tool, clearly showed the start of the machining unstable conditions that produce the chatter phenomenon. With respect to the workpiece, the accelerometer showed vibrations that grew gradually as chatter arouse, but the transition state was not as evident as for the displacement sensor.

A frequency analysis of both sensors was conducted. Exciting frequencies produced by the tool vibrations are related to the spindle rotational speed. These frequencies can be extracted from the inductive sensor measurements. However, the workpiece-table system natural frequencies are not known, so an impact test was performed to obtain this information. Figure 3 shows the results of this test. Two major frequencies located at 225 and $476 \mathrm{~Hz}$ can be seen, and one of them has been labeled as the workpiece main frequency (WF) for analysis purposes.

Results of the frequency analysis for the tool vibrations can be observed as shown in Figure 4. The characteristic frequencies for the three major regions were plotted. For the chatter-free machining state, only the spindle frequency (SF) was present. This is due to the unbalance of the tool-spindle system. The spindle frequency corresponded to the cutting conditions established for this 


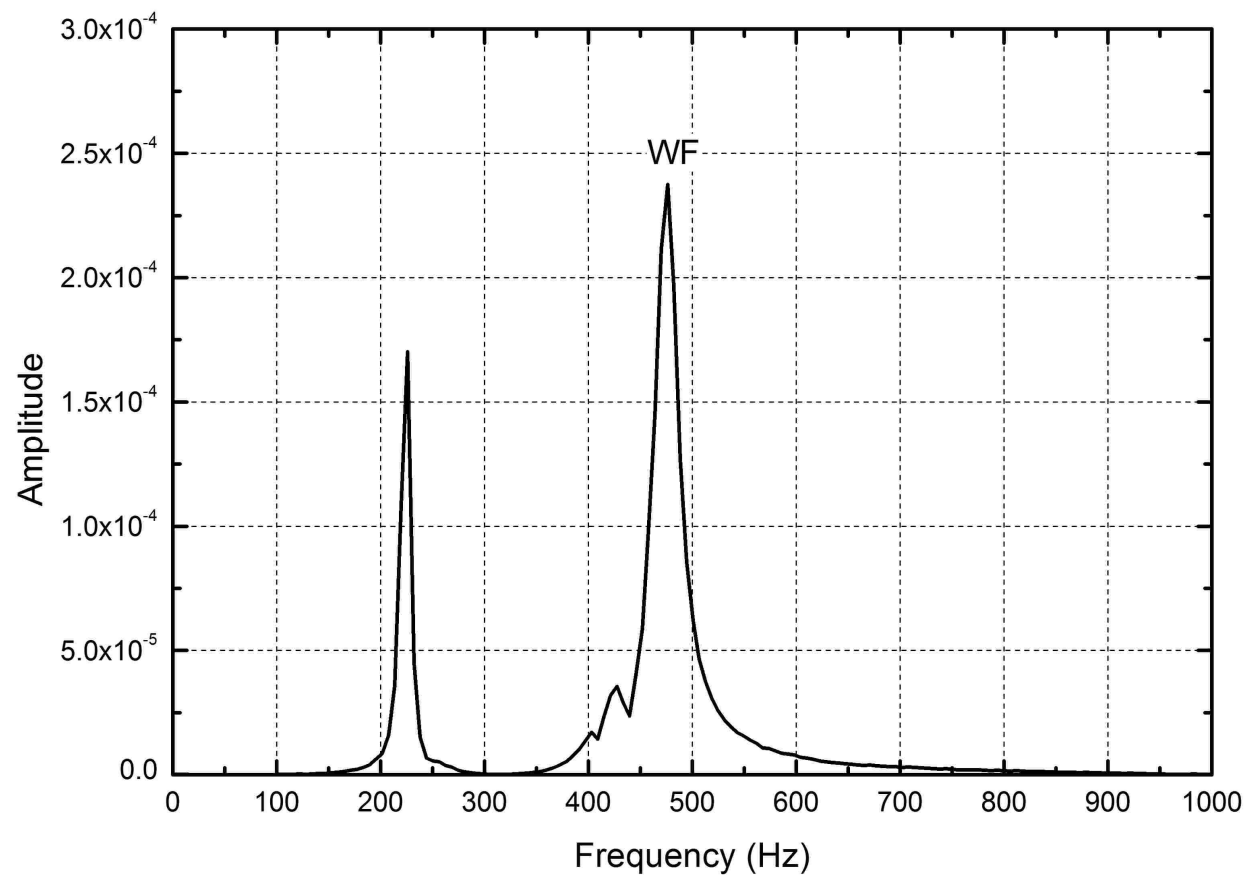

Figure 3. Impact test results for the workpiece-table system.
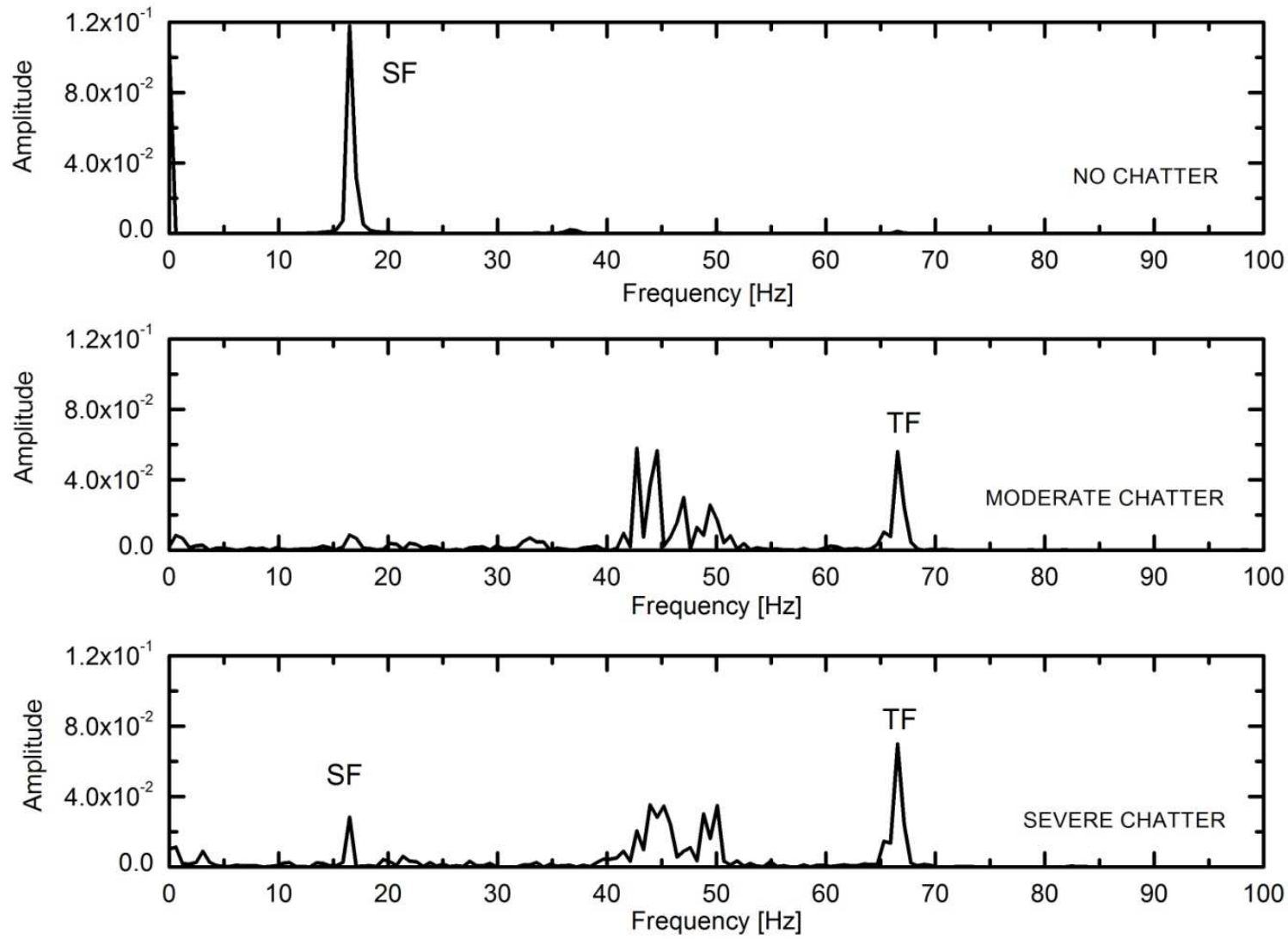

Figure 4. Frequency analysis of the tool vibrations measured with the inductive sensor. 

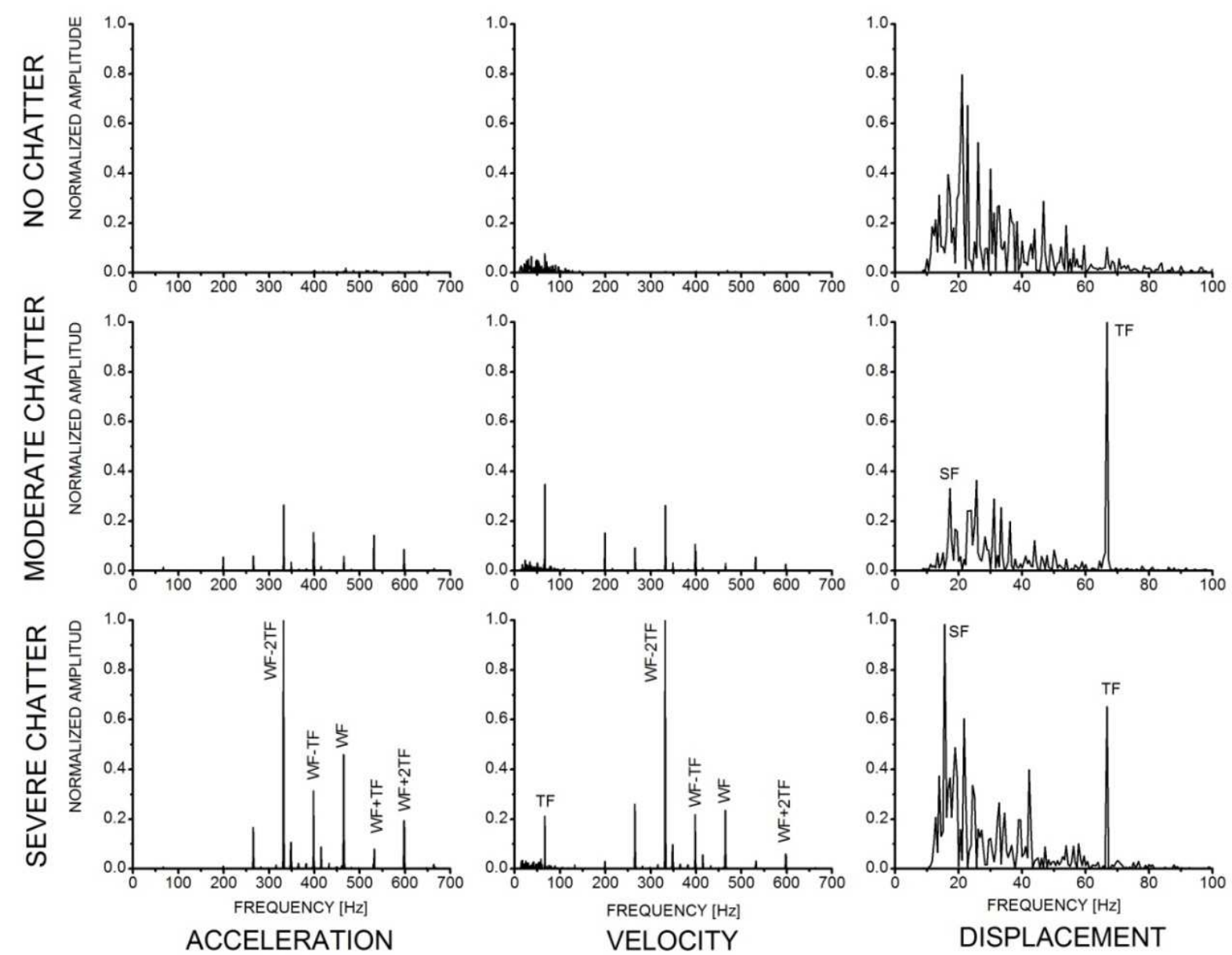

Figure 5. Vibrations analysis by variable type and machining conditions, measured at the workpiece.

test $(1000 \mathrm{rpm})$. When chatter occurred, the spindle frequency was attenuated, but the tool vibrated at the tooth passing frequency (TF) of $66.66 \mathrm{~Hz}$. An additional modulation phenomenon produced by SF and TF was present between 40 and $50 \mathrm{~Hz}$. These results, with well localized frequencies, were in contrast with those found by Kuljanic et al. (2008), who show frequency spectra for force measurements at the spindle with harmonics within all the range of the frequencies plotted.

The velocity of the vibrations is the most common parameter used in predictive maintenance and various conversion techniques are used to obtain this parameter from acceleration measurements. To analyze the relevance of the different vibrations measurement types, named acceleration, velocity and displacement, in relation to the information that each parameter offers for chatter identification, the three regions as shown in Figure 2 were analyzed separately. Figure 5 shows the resulting spectra for the three vibration variables under different machining operating conditions. Graphs were normalized by column, and amplitudes can be compared vertically for each variable. Since the acceleration of motion emphasizes high frequencies, the low frequencies produced by the spindle rotation and tooth impacts were not appreciated in the acceleration graphs (TF, SF). However, high frequencies of the workpiece-structure system excited by the tool were evident for this variable when chatter was present (WF). Furthermore, the displacement of motion emphasizes low frequencies, so when the acceleration signal was converted to displacement, the higher frequencies (WF) were attenuated in such a way that their amplitudes were not comparable to the tool vibrations amplitude (TF, SF). The frequency scale was limited to $100 \mathrm{~Hz}$ for this variable for a better appreciation of the lower frequencies.

In the absence of chatter, there was no clear definition of vibration peaks in the spectra. However, for moderate and severe chatter, the high frequencies measured at the workpiece were evident in the acceleration signal (WF), while the excitation frequencies produced by the toolspindle system (TF, SF) were also evident in the displacement signal at this measurement point.

With respect to the velocity signal, when severe chatter was present, the frequency produced by the tooth 
impacts was clearly observed, as well as the higher frequencies corresponding to the workpiece-structure vibrations. Even in the case of moderate chatter, the velocity signal showed the low and high frequency components. There are reports of force measurements at the table for milling where cutting and chatter frequencies can be observed in the frequency spectra (Suh et al., 2002). An additional effect can be observed in Figure 5 in the acceleration and velocity signals for moderate and severe chatter, where a frequency modulation resulted in the combined effects of the workpiece-structure and tool vibrations (WF $\pm n T F)$. There are also reports of modulation effects due to the chip thickness variation (Toh, 2004).

Inductive and accelerometer frequency spectra results were characterized by well localized vibration peaks, in accordance with Liao and Young (1996). They showed similitude in that minor changes could be observed in TF when chatter was developing from the transition state to the severe chatter state. However, chatter frequencies, treated as the workpiece frequencies excited by the tool impacts, showed a clear trend to grow in amplitude in every stage of chatter development. Additionally, TF can be used as another evidence of the unstable machining condition when using velocity measurements. From these results, it can be established that the velocity signal can be useful to detect the development of chatter in machining operations.

\section{Conclusions}

This work has been conducted to analyze the chatter development in milling operations from tool and workpiece vibrations analyses. The tool vibrations were measured with an inductive sensor and the workpiece with an accelerometer. It was found that frequencies are well localized in the spectra and are in accordance with the exciting and natural frequencies of the spindle and workpiece-table system, respectively. Acceleration measurements at the workpiece attenuate spindle frequencies, while displacement measurements at the same measurement point attenuate the high frequency peaks of chatter. Velocity measurements, obtained from acceleration data, are good indicators of chatter development, because the frequency spectra show the spindle and workpiece vibrations adequately.

\section{REFERENCES}

Axinte DA, Gindy N, Fox K, Unanue I (2004). Process monitoring to assist the workpiece surface quality in machining. Int. J. Mach. Tools Manuf. 44:1091-1108

Albrecht A, Park SS, Altintas Y, Pritschow G (2005). High frequency bandwidth cutting force measurement in milling using capacitance displacement sensors. Int. J. Mach. Tools Manuf. 45:993-1008.

Bagci E (2011). Monitoring and analysis of MRR-based feedrate optimization approach and effects of cutting conditions using acoustic sound pressure level in free-form surface milling. Sci. Res. Essays 6:256-277.

Insperger T, Stépán G, Bayly PV, Mann BP (2003). Multiple chatter frequencies in milling processes. J. Sound Vib. 262:333-345.

Khraisheh MK, Pezeshki C, Bayoumi E (1995). Time series based analysis for primary chatter in metal cutting. J. Sound Vib. 180:67-87.

Kuljanic E, Sortino M, Totis G (2008). Multisensor approaches for chatter detection in milling. J. Sound Vib. 312:672-693.

Liao YS, Young YC (1996). A new on-line spindle speed regulation strategy for chatter control. Int. J. Mach. Tools Manuf. 36: 651-660.

Ryabov O, Mori K, Kasashima N (1998). Laser displacement meter application for milling diagnostics. Opt. Lasers Eng. 30:251-263.

Schmitz TL, Smith KS (2012). Mechanical vibrations: modeling and measurement. ISBN: 978-1-4614-0459-0. Springer New York, Dordrecht Heidelberg London.

Suh CS, Khurjekar PP, Yang B (2002). Characterization and identification of dynamic instability in milling operation. Mech. Syst. Signal Pr. 16:853-872.

Tang WX, Song QH, Yu SQ, Sun SS, Li BB, Du B, Ai X (2009). Prediction of chatter stability in high-speed finishing end milling considering multi-mode dynamics. J. Mater. Process. Technol. 209:2585-2591.

Tatar K, Gren P (2008). Measurement of milling tool vibrations during cutting using laser vibrometry. Int. J. Mach. Tool Manu. 48:380-387.

Toh CK (2004). Vibration analysis in high speed rough and finish milling hardened steel. J. Sound Vib. 278:101-115.

Tsai NC, Chen DC, Lee RM (2010). Chatter prevention for milling process by acoustic signal feedback. Int J. Adv. Manuf. Technol. 47:1013-1021. 